\title{
Com quem vou ao médico? Um estudo com homens da zona Sul de São Paulo
}

RESUMO | O objetivo do estudo foi conhecer com quem os homens comparecem aos serviços de saúde e quem seriam os acompanhantes. O cenário da pesquisa foi um ambulatório localizado na zona sul da cidade de São Paulo e contou com a participação de 51 indivíduos. Tratou-se de um estudo exploratório e descritivo, com abordagem quanti-qualitativa. A coleta dos dados foi feita por meio de um questionário semiestruturado. Os dados numéricos foram organizados em temas, agrupados em tabelas. As informações foram analisadas utilizando-se o método do Discurso do Sujeito Coletivo. Os resultados mostraram que $65 \%$ dos homens comparecem sós ao serviço, $27 \%$ citaram a esposa, destacando a parceria existente, $4 \%$ mencionaram a mãe, denotando vínculo materno, e $4 \%$ declararam a presença dos filhos. Constata-se que os homens vêm assumindo o protagonismo nos seus cuidados. Mesmo diante de um trabalho incipiente, vislumbra-se uma possibilidade de mudança.

Palavras-chaves: Saúde do homem; Masculinidades; Promoção da Saúde.

ABSTRACT | The aim of the study was to find out with whom men attend health services and who would be the companions. The research scenario was an outpatient clinic located in the south of the city of São Paulo and had the participation of 51 individuals. It was an exploratory and descriptive study, with a quantitative and qualitative approach. Data collection was performed using a semi-structured questionnaire. The numerical data were organized into themes, grouped in tables. The information was analyzed using the Collective Subject Discourse method. The results showed that $65 \%$ of men attend the service alone, $27 \%$ mentioned the wife, highlighting the existing partnership, $4 \%$ mentioned the mother, denoting a maternal bond, and $4 \%$ declared the presence of their children. It appears that men have assumed the leading role in their care. Even in the face of incipient work, there is a possibility of change.

Keywords: Men's Health; Masculinity; Health Promotion

RESUMEN | El objetivo del estudio fue conocer con quiénes acuden los hombres a los servicios de salud y quiénes serían los acompañantes. El escenario de investigación fue un ambulatorio ubicado en el sur de la ciudad de São Paulo y contó con la participación de 51 personas. Fue un estudio exploratorio y descriptivo, con enfoque cuantitativo y cualitativo. La recolección de datos se realizó mediante un cuestionario semiestructurado. Los datos numéricos se organizaron en temas, agrupados en tablas. La información se analizó mediante el método del Discurso Colectivo del Sujeto. Los resultados mostraron que el $65 \%$ de los hombres asisten solos al servicio, el $27 \%$ mencionó a la esposa, destacando la asociación existente, el $4 \%$ mencionó a la madre, denotando vínculo materno, y el $4 \%$ declaró la presencia de sus hijos. Parece que los hombres han asumido el papel principal en su cuidado. Incluso ante un trabajo incipiente, existe la posibilidad de cambio.

Palabras claves: Salud del Hombre; Masculinidad; Promoción de la salud.

\section{Maria Cecília Leite de Moraes}

Doutora em Saúde Pública pela Faculdade de Saúde Pública da Universidade de São Paulo. Pós Doutora pela Escola de Enfermagem da Universidade Federal da Bahia, Pesquisadora do Grupo de Estudos da Criança e do Adolescente-CRESCER da EFJUFBA. ORCID: 0000-0002-8717-6513

\section{Sirlei Aparecida Novato Hodge}

Enfermeira, Pós-graduada em Saúde Pública pelo Centro Universitário Adventista de São Paulo.

ORCID: 0000-0001-7517-4484

\section{Robson da Costa Oliveita}

Enfermeiro, Mestre em Promoção da Saúde pelo Centro Universitário Adventista de São Paulo. Professor da Universidade Ibirapuera. ORCID: 0000-0001-6532-2893

Recebido em: 11/12/2020

Aprovado em: 08/01/2021

\section{Climene Laura de Camargo}

Doutora em Saúde Pública pela Faculdade de Saúde Pública da Universidade de São Paulo, Pós Doutora pela Universidade René Descartes, Paris V, Sorbonne; Professora da Escola de Enfennagem da Universidade Federal da Bahia, Coordenadora e !Pesquisadora do Grupo de Estudos da Criança e do Adolescente-CRESCER da EFJUFBA.

ORCID: 0000-0002-4880-3916

\section{INTRODUÇÃO}

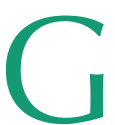
ênero distingue a diferença entre masculino e feminino, marcando também um constructo referente a padrões de condutas $^{(1)}$. Além do que, caracteriza as relações entre os dois grupos, fator que mobiliza, de maneira categórica, vários aspectos da vida. A partir de tal afirmação, é possível inferir que a observação do estado e cuidado da saúde inclui a questão de gênero ${ }^{(1)}$.

As fragilidades são atribuídas às muIheres. Concomitantemente, existe uma relação histórica entre cuidado e a saúde feminina, que observa, especialmente, a saúde sexual e reprodutiva, fato que naturalizou essa atuação ${ }^{(2)}$. De maneira oposta, indivíduos do sexo masculino possuem uma grande dificuldade em aceitar suas vulnerabilidades ${ }^{(3)}$. A denegação da doença, do sofrimento e da dor são uma realidade entre sujeitos deste grupo. Tal circunstância contribui para realçar as frequentes mortes por causas evitáveis ${ }^{(4)}$.

Em tempos recentes, para elucidar as assertivas mencionadas, estudiosos incorporaram o conceito de masculinidade ${ }^{(5-6)}$ aos estudos relacionados a gênero. Esta concepção corresponderia aos papéis e relações entre o masculino e o femini- 
no e às práticas com as quais indivíduos dos dois grupos se pactuam com seus lugares sociais. Abrangem, também, as repercussões desses papéis na personalidade e cultura. Entretanto, admite-se que os estudos comparativos de gênero se mostram inapropriados para explicitar os comportamentos na busca por ajuda ${ }^{(7)}$.

A afirmação da masculinidade encobre a autopercepção sobre o corpo, coadjuvando para que os homens desconheçam o seu estado de saúde, cuidem-se menos ou, simplesmente, não se cuidem $^{(8-9)}$. Ademais, cita-se que a alteração do entendimento na percepção da necessidade de saúde interfere na busca por assistência ${ }^{(1)}$. Ratifica-se que sujeitos do sexo masculino procuram por socorro quando em estado crítico de saúde ${ }^{(10)}$.

Outrossim, o constructo mencionado estimula a automedicação; além do que, fomenta as ações curativas em detrimento das preventivas ${ }^{(11)}$. Os homens não são proativos na atenção à saúde, na procura por serviço médico ou no aconselhamento no que se refere à promoção de saúde ${ }^{(12)}$.

Abre-se um adendo para lembrar que a preocupação com os hábitos e cuidados sanitários masculinos fez com que fosse criada a Política Nacional de Atenção Integral à Saúde do Homem (PNAISH) no ano de 2009; fato que representou uma importante inovação neste campo $^{(13)}$. Dentre as prioridades estabelecidas, privilegiaram-se trazer esta coletividade para os cuidados de sua saúde e assinalar à população geral sobre a realidade singular masculina ${ }^{(14)}$.

Os serviços, por vezes, não dialogam com o PNAISH. E é lícito afirmar que, ainda no presente, os sistemas não distinguem as peculiaridades dos sujeitos ${ }^{(15)}$. O formato do acolhimento e a prestação dos serviços não diferenciam os homens, aspecto que retardaria os avanços pretendidos para a sociedade ${ }^{(1)}$. Vale destacar que pesquisas recentes mostram maior mortalidade e menor expectativa de vida entre a população masculina, fatores que se associam a questões contextuais ${ }^{(16)}$.
Ao mesmo tempo, trabalhos contemporâneos mostram algumas sutis transformações nas atitudes masculinas, fator que repercute positivamente nos cuidados de saúde ${ }^{(3)}$. Há indicativos de melhora no que diz respeito ao monitoramento da saúde.

Observando o cenário descrito, considerou-se oportuno compreender aspectos do cuidado de saúde masculino. Assim, a finalidade do estudo foi conhecer como se processa o comparecimento do homem ao serviço de saúde: se só ou acompanhado.

\section{MÉTODO}

Este artigo foi estruturado a partir da pergunta: "com quem você vem ao serviço de saúde?", um dos tópicos da pesquisa "Conhecendo atitudes masculinas". Tratou-se de um estudo de campo, exploratório e descritivo, abordagem quanti-qualitativo, de corte transversal.

A pesquisa foi realizada nas dependências do Posto Avançado do Hospital Adventista de São Paulo (serviço ambulatorial), localizado no bairro do Capão Redondo, na região Sul da cidade de São Paulo.

A amostra de conveniência foi constituída por 51 indivíduos do sexo masculino, maiores de dezoito anos de idade. As informações foram obtidas por meio de um questionário formulado para a pesquisa. O instrumental foi respondido pelo entrevistado ou pelo pesquisador, quando necessário.

Todos os participantes assinaram o Termo de Consentimento Livre e Esclarecido. A pesquisa foi aprovada pelo
Comitê de Ética em Pesquisa (CEP) do Centro Universitário Adventista de São Paulo - UNASP, sob o número 2.381.684. A coleta dos dados ocorreu entre os meses de outubro e dezembro de 2017.

As justificativas das respostas sobre a presença, ou não, de acompanhante estão dispostas em forma discursiva, já que as respostas foram analisadas por meio da técnica do Discurso do Sujeito Coletivo (DSC). Tal método permite tabular e organizar materiais provenientes de depoi$\operatorname{mentos}^{(17)}$.

O método do DSC contempla os seguintes procedimentos: seleção de expressões-chave $(\mathrm{ECH})$ extraídas de cada resposta dada pelos participantes; identificação da ideia central (IC) presente na expressão-chave e identificação das ideias centrais semelhantes ou complementares. A reunião das expressões-chave com ideias centrais semelhantes ou complementares são agregadas em um único discurso, que constitui a concepção coletiva ${ }^{(18)}$. O Discurso do Sujeito Coletivo (DSC) é o manifesto do discurso síntese.

As respostas sobre a existência e identificação de acompanhante ao serviço são apresentadas em tabelas compostas por números e porcentagens.

\section{RESULTADOS}

A seguir são apresentados os resultados obtidos pela pesquisa. As tabelas mostram os números e as porcentagens de respostas obtidas.

Os dados da tabela 1 destacam a ida solitária $(64,7 \%)$ ao serviço de saúde.
Tabela 1: Número e porcentagem de respostas sobre a presença de acompanhantes - São Paulo, 2017

$\begin{array}{lcc}\text { A. Sem acompanhante (só) } & 33 & 64,7 \\ \text { B. Com acompanhante } & 17 & 33,3 \\ \text { C. Indiferente } & 1 & 2,0 \\ \text { Total } & 51 & 100,0\end{array}$

Fonte: Pesquisa "Conhecendo atitudes masculinas" - 2017 
Dentre os acompanhantes, as respostas evidenciam a presença da esposa (53\%).

Os núcleos temáticos apresentam as categorias discursivas obtidas na pesquisa. É interessante observar que os indivíduos que compareceram sós ao serviço de saúde destacaram apenas o fato de estarem desacompanhados.

\section{Núcleo temático: Ida solitária}

\section{IC: Sempre só}

DSC: Quando vou ao médico, sempre vou sozinho.

Dentre os acompanhantes, ressalta-se a presença da esposa (53\%) como acompanhante.

Núcleo temático: Presença da esposa

IC: Dependência e parceria

DSC: Só venho com minha esposa, sem ela não sei o que fazer. Acho muito importante ela saber como eu estou.

Cabe denotar os relatos da presença materna como acompanhante (11,7\%), haja vista que a pesquisa aconteceu entre a população adulta.

\section{Núcleo temático: Presença da mãe}

\section{IC: Cuidado materno}

DSC: Vou com minha mãe, se minha mãe não me fala, nem venho....

De maneira antagônica, outros 11,7\% dos respondentes apontaram o filho como acompanhante.

Núcleo temático: Presença do fillho

IC: Sistema de cuidado filial

DSC: Vou sempre acompanhado, meu filho vai sempre junto comigo.

No estudo, quatro dos entrevistados
$(23,6 \%)$ não referiram a pessoa que os acompanhava. Entretanto, foi revelado que a presença do acompanhante estava relacionada à sua condição.

Núcleo temático: Paciente indeterminado

IC: Fragilidade

DSC: Só venho sempre acompanhado porque sinto-me frágil.

Apenas um elemento do grupo $(2 \%)$ relatou que a frequência ao serviço de saúde independe da presença de um acompanhante.

Núcleo temático: Indiferente

IC: Vou de qualquer maneira

DSC: Se não tiver quem venha, vou só.

\section{DISCUSSÃO}

Chamou a atenção o número de homens que procura o serviço de saúde desacompanhado A ida solitária aos serviços de saúde pode estar associada ao medo de expor dores e sofrimentos. Pesquisadores $^{(11)}$ evidenciam que os homens reprimem suas necessidades de saúde, assim como apresentam dificuldades de expressá-las. Estes aspectos talvez expliquem o fenômeno de estar só no serviço de saúde. Outro constatou que o homem procura resolver seus problemas de saúde de forma prática ${ }^{(19)}$. Tal afirmação reforça mudanças no comportamento masculino ${ }^{(3)}$.

A esposa como acompanhante distingue a cumplicidade que se instala ao estar junto e valoriza a presença feminina nos cuidados de saúde do homem. Além do que, a mulher é um robusto ator social e

Tabela 2: Número e porcentagem de respostas sobre quem são os acompanhantes - São Paulo, 2017

\begin{tabular}{lccc} 
& Categorias & N & $\%$ \\
A. Esposa & 9 & 53,0 \\
\hline B. Mãe & 2 & 11,7 \\
C. Filho & 2 & 11,7 \\
\hline D. Não discriminou & 4 & 23,6 \\
\hline Total & 17 & 100,0
\end{tabular}

Fonte: Pesquisa "Conhecendo atitudes masculinas" - 2017 uma das principais referências na atenção familiar ${ }^{(20-10)}$, assumindo o protagonismo nestas situações ${ }^{(21)}$. Guarda um espaço singular, supervisionando, atuando, tomando decisões, acompanhando e avaliando a saúde e a doença do homem.

Em face das dificuldades dos homens em procurar a assistência de saúde, observa-se a importância do papel materno, contribuindo para que muitos indivíduos só cheguem ao serviço acompanhados pela mãe $\mathrm{e}^{(19)}$. Denota-se, uma vez mais, que as mulheres concentram a responsabilidade pela diligência família, independentemente de sua idade e classe social ${ }^{(22)}$. E, quando figura materna, agrega e articula os cuidados dos filhos, em diferentes circunstâncias, principalmente nos casos de doença ${ }^{(20)}$. Assim, observa-se que o papel materno contempla a coordenação da saúde que abrange desde a supervisão das rotinas até o acompanhamento ao serviço médico ${ }^{(23)}$.

Observou-se a responsabilidade dos filhos nos cuidados dos pais, exercendo, muitas vezes, o papel de cuidador primário, quer por questões financeiras, quer por questões afetivas ${ }^{(24)}$. Ademais, tal fato costuma acontecer quando o cônjuge é falecido ou não apresenta condição para desempenhar a tarefa. A presença do familiar, subsidia o sistema de cuidados $2 \underline{(23)}$, traz motivação à realização do tratamento, visa o conforto e busca uma possível recuperação ${ }^{(25)}$. Estudo anterior discorre sobre as obrigações emergentes na cultura familiar, na qual prevalece o dever da retribuição pelos cuidados do passado ${ }^{(26-27)}$.

O discurso da fragilidade expõe vários aspectos da masculinidade. Exibe, de maneira contundente, a limitação masculina no que diz respeito ao sofrimento; traço encoberto nos aspectos históricos e peculiares do gênero ${ }^{(3-19-28)}$. Aponta, ainda, a obrigatoriedade do acompanhamento, fato que pode estar associado à dificuldade em expor vulnerabilidades ${ }^{(29)}$. Uma pessoa de confiança, um mediador, pode facilitar o contato do homem com o serviço de saúde.

A concepção de atenção à saúde independentemente de estar acompanhado 
é consonante com os resultados de uma pesquisa recente, na qual emerge a figura do homem autônomo no que diz respeito ao autocuidado ${ }^{(2)}$.

\section{CONCLUSÕES}

Ainda em dias atuais, distingue-se a vigorosa participação da mulher na atenção da saúde masculina, seja como esposa, seja como mãe. Elas acompanham os homens em seus cuidados, visto que apenas um elemento do grupo
( $2 \%)$ relatou comparecer ao serviço de saúde independentemente da presença de acompanhante.

O núcleo da assistência se consolida na presença dos filhos como acompanhantes; aspecto que reforça a importância da presença familiar tanto para o monitoramento da saúde, como para o enfrentamento de doenças.

Mesmo diante da tradição conhecida sobre a questão do cuidado da saúde masculina, a presença de homens desacompanhados aos serviços trouxe uma importante referência, que pode evidenciar a relação entre a ida solitária aos serviços de saúde e o medo de expor dores e sofrimentos, condição esta que toma corpo pelo expressivo número encontrado: $64,7 \%$.

Em face de resultados considerados incipientes, é possível vislumbrar um realinhamento ou uma nova tipologia no que diz respeito ao cuidado de saúde masculino. Serão necessárias mais pesquisas para a confirmação dos achados, contudo o futuro parece auspicioso.

\section{Referências}

1. Tavares VMC, Neto LMA, dos Santos Pereira E, Taveira MDGMM, Cavalcante JK, \& Correia DS. Roda de conversa: atenção integral à saúde das mulheres e questões de gênero. Brazilian Journal of Development. 2020;6(8): 61501-61510. 2. Barata RB. Como e por que as desigualdades sociais fazem mal à saúde. Rio de Janeiro: Editora Fiocruz; 2009.

3. Leite de Moraes MC, da Costa Oliveira R, Silva MJ. Uma questão masculina: conhecendo possíveis entraves para a realização dos exames de detecção do câncer de próstata. Revista Médica Herediana. 2017;28(4):230-235.

4. Botton, Andressa; Cúnico, Sabrina Daiana; Strey, Marlene Neves. Diferenças de gênero no acesso aos serviços de saúde: problematizações necessárias. Mudanças-Psicologia da Saúde. 2017;25(1):67-72.

5. Sealey R, George N, Gordon S, Simmons L. Dual Benefits of a Student-Assisted Interprofessional Men's Healthy Lifestyle Pilot Program. American Journal of Mens Health. 2017;11(4):1133-1141.

6. Separavich MA, Canesqui AM. Saúde do homem e masculinidades na Política Nacional de Atenção Integral à Saúde do Homem: uma revisão bibliográfica. Saúde e Sociedade. 2013;22(2):415-428.

7. Sousa AR, Queiroz AM, Florencio RMS, Portela PP, Fernandes JD, Pereira A Homens nos serviços de atenção básica à saúde: repercussões da construção social das masculinidades. Revista Baiana de Enfermagem. 2016;30(3):1-10.

8. Sach TH, Whynes DK. Men and women: beliefs about cancer and about screening. BMC Public Health. 2009;24(9):431.

9. Machin R, Couto MT, Silva GSND, Schraiber LB, Gomes R, Santos Figueiredo WD, Pinheiro TF. Concepções de gênero, masculinidade e cuidados em saúde: estudo com profissionais de saúde da atenção primária. Ciência Saúde Coletiva. 2011; 16:4503-12.

10. Leite de Moraes MC, Costa Oliveira R, Jesus MS. A elaboração de um folder sobre câncer da próstata. The preparation of a folder about prostate cancer. La elaboración de un folder sobre cáncer de la próstata. Rev. Fac. Cienc. Salud. UDES (Bucaramanga). 2018;5(2):19-24.

11. Novato $S$, Leite de Moraes MC. Perfil de homens que procuram um serviço de saúde na região sul do município de São Paulo. Interfaces Científicas. 2020; 8(3):66-75.

12. Schraiber LB, Figueiredo WDS, Gomes R, Couto MT, Pinheiro TF, Machin $R$, Valença, 0 . Necessidades de saúde e masculinidades: atenção primária no cuidado aos homens. Cadernos de Saúde Pública. 2010;26:961-70.

13. Bollard M. Health promotion and intellectual disability: listening to men. Health Soc Care Community. 2017;25(1):185-193.

14. Ministério da Saúde (BR). Política Nacional de Atenção Integral à Saúde do Homem: princípios e diretrizes. 2008:01-40.

15. Aguiar RS, Santana DC, Santana PC. A percepção do enfermeiro da estratégia saúde da família sobre a saúde do homem. Revista de Enfermagem do Centro-Oeste Mineiro. 2015;set.-dez.5(3):1844-1854.

16. Gomes TMF, Santos GSJ, de Assis, EV. Ações na atenção à da saúde do homem: revisão integrativa da literatura. Journal of Medicine and Health Promotion. 2018; 3(4):1063-72.

17. Galdas PM, Cheater F, Marshall P. Men and health help-seeking behaviour: literature review. J Adv Nurs. 2005;Mar.49(6):616-23.

18. Lefèvre $F$, Lefèvre AMC, Teixeira JJV. 0 discurso do sujeito coletivo: uma nova abordagem metodológica em pesquisa qualitativa. Caxias do Sul: EDUCS. 2000: 138p.

19. Lefèvre $F$, Lefèvre AMC, Cornetta VK, Teixeira de Araújo SD. 0 discurso do sujeito coletivo como eu ampliado: aplicando a proposta em pesquisa sobre a pilula do dia seguinte. Journal of Human Growth and Development.2010;20(3):798-808.

20. Toneli MJF, Souza MGC, Müller RCF. Masculinidades e práticas de saúde: retratos da experiência de pesquisa em Florianópolis/SC. Physis: Revista de Saúde Coletiva. 2010;20(3):973-994.

21. Silva MM da, Budó MLD, Resta DG, Silva SO, Ebling SBD, Carvalho SORM. Integralidade na saúde da família: limites e possibilidades na perspectiva da equipe/The entire family health: limits and possibilities in view of the team. Ciência, Cuidado e Saúde. 2013;12(1):155-163.

22. SANTOS-ORLANDI, Ariene Angelini dos et al. Perfil de idosos que cuidam de outros idosos em contexto de alta vulnerabilidade social. Escola Anna Nery. 2017;21(1):2017.

23. Sousa LP, Guedes DR. A desigual divisão sexual do trabalho: um olhar sobre a última década. Estudos Avançados. 2016;30(87):123-139.

24. SOUSA QUEIROZ, Talita et al. Como homens idosos cuidam de sua própria saúde na atenção básica?. Revista Brasileira de Enfermagem. 2018;71.

25. Pocinho R, Belo P, Melo C, Navarro-Pardo E, Muñoz JJF. Relação entre 0 estado psicossocial do cuidador informal e o tempo de cuidado dos idoso da região centro de Portugal. Educación y Humanismo. 2017;19(32):88-101. 26. Sehn AC, Braz MEE, Rozeno RF. A FAMÍLIA COMO UNIDADE DE CUIDADOS NA SAÚDE E NA DOENCA. RSDA [Internet]. $17^{\circ}$ de junho de 2019 [citado $8^{\circ}$ de janeiro de 2021];3(1):54-1. Disponível em: http://revista.domalberto.edu. br/index.php/revistadesaudedomalberto/article/view/411Fomatos de Citação 27. Albano BR, Basílio MC, Neves JB. Desafios para a inclusão dos homens nos serviços de atenção primária à saúde. Revista Enfermagem Integrada. 2010;3( 2): $554-563$.

28. Aires M, Pizzol FLFD, Bierhals CCBK, Mocellin D, Fuhrmann AC, Santos NOD, et al. Responsabilidade filial no cuidado aos pais idosos: estudo misto. Acta Paulista de Enfermagem. 2019;32(6):691-699.

29. Abrahão F, Oliveira RDC, Moraes MCLD, Sousa ARD. Homens imigrantes bolivianos residentes na zona central do município de São Paulo: situação de moradia e saúde. Revista de Divulgação Científica Sena Aires. 2020;9(1):97-108. 30. Carrara S, Russo JA, Faro L. A política de atenção à saúde do homem no Brasil: os paradoxos da medicalização do corpo masculino. Physis: revista de saúde coletiva. 2009;19(3):659-678. 